\title{
Development of composite road environment safety index
}

\begin{abstract}
This study aims to develop a road safety index that combines selected road environmental characteristics in Malaysia. Firstly, 14 indicators that generally portray the Malaysian road environments were selected. Then, the final list of specific indicators for each road network was developed. The indicators were derived based on the specific criteria such as the objectives, method of measurement, quality, and expected outcomes of the indicators that may improve the overall road safety of the Malaysian trunk roads. The Malaysian Federal Road 12 was selected as the study area to assess the applicability of the theory. By employing the principal component analysis, four components were obtained and from the statistical weightage of the indicator in each component, the composite indexes were calculated. The results showed that the sections with low number of reported crashes were not necessarily safe for road users. Instead, poor road environment conditions may be highly hazardous to road users. The presence of heavy vehicles and motorcycles was found to be the main risk factor of crash occurrences on this road. Overall, the crash data may be supplemented with another proactive method in order to get a broader picture of the poor road sections.
\end{abstract}

Keyword: Composite road environment; Crash data; Road safety ranking; Road section 